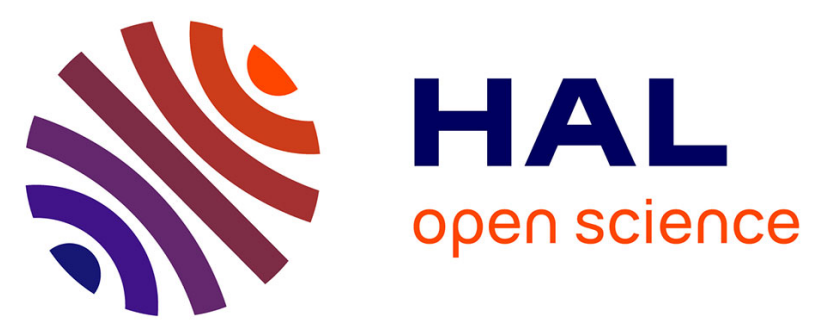

\title{
Scaled Growth of an Isolated Droplet Generated by Laser-Induced Phase Separation in a microemulsion - Comparison with Systems of the Same Ising Class
}

C. Lalaude, Jean-Pierre Delville, Yves Garrabos, Eric Freysz, A. Ducasse

\section{- To cite this version:}

C. Lalaude, Jean-Pierre Delville, Yves Garrabos, Eric Freysz, A. Ducasse. Scaled Growth of an Isolated Droplet Generated by Laser-Induced Phase Separation in a microemulsion - Comparison with Systems of the Same Ising Class. Journal de Physique IV Proceedings, 1995, 05 (C3), pp.C3-267-C3-272. 10.1051/jp4:1995325 . jpa-00253691

\section{HAL Id: jpa-00253691 \\ https://hal.science/jpa-00253691}

Submitted on 1 Jan 1995

HAL is a multi-disciplinary open access archive for the deposit and dissemination of scientific research documents, whether they are published or not. The documents may come from teaching and research institutions in France or abroad, or from public or private research centers.
L'archive ouverte pluridisciplinaire HAL, est destinée au dépôt et à la diffusion de documents scientifiques de niveau recherche, publiés ou non, émanant des établissements d'enseignement et de recherche français ou étrangers, des laboratoires publics ou privés. 


\title{
Scaled Growth of an Isolated Droplet Generated by Laser-Induced Phase Separation in a microemulsion - Comparison with Systems of the Same Ising Class
}

\author{
C. Lalaude, J.P. Delville, Y. Garrabos*, E. Freysz and A. Ducasse \\ Centre de Physique Moléculaire Optique et Hertzienne, URA 283 du CNRS, Université Bordeaux I, \\ 33405 Talence cedex, France \\ * Laboratoire de Chimie du Solide UPR 8661 du CNRS, Université Bordeaux I, 33405 Talence cedex, \\ France
}

\begin{abstract}
Résume: Nous présentons et analysons la croissance d'une goutte isolée engendrée par une trempe locale en composition induite par laser dans une microémulsion. La dynamique de cette croissance produite dans un capillaire, et donc soumise à des contraintes optique et spatiale, est étudiée dans le cadre plus général de la cinétique de séparation de phase dans les systèmes appartenant à la classe d'universalité $d=3, n=1$ du modéle Ising. Celle-ci peut être analysée avec deux variables réduites sans dimension définies à partir d'une échelle de longueur et de temps caractéristiques dépendant du système. Les dynamiques observées de ces systèmes sont ici comparées dans un même référentiel. Aux temps longs, il apparait que le comportement expérimental de croissance en $(\tau)^{1 / 3}$ ( $\tau$ étant l'échelle de temps réduite) correspond au mécanisme le plus lent observé aussi bien pour une assemblée de gouttes que pour une goutte isolee.
\end{abstract}

\begin{abstract}
We present and analyze the growth of an isolated droplet generated by a laser-induced local quench in composition in a microemulsion. The growth dynamics realized in a capillary and thus under optical and spatial constraints is studied in the most general frame of the phase separation kinetics in systems belonging to the universality Ising class $d=3, n=1$. It can be analyzed with two dimensionless reduced variables defined from two characteristic length and time scales of the system. The observed dynamics of these systems are here compared using the same scaled parameters. In the late stage, it appears that the experimental growth behavior in $(\tau)^{1 / 3}$ ( $\tau$ being the reduced time) corresponds to the slowest kinetics mechanism for a droplets assembly as well as for an isolated droplet.
\end{abstract}

\section{INTRODUCTION}

The dynamics of phase separation in critical systems is a difficult problem whose various aspects are still unresolved. The case of systems belonging to the universality class $d=3, n=1$ of the Isingmodel where $d$ and $n$ are respectively space and order parameter dimensionalities [1], is indeed the more commonly studied. It has been the object of important theoretical and experimental works during the last ten years[2-13]. One important goal of these studies is the determination of universal growth laws describing the dynamics of the induced phase transition. In order to compare the dynamical behavior of the various analyzed systems, the experimental data are usually discussed in terms of dimensionless reduced variables in which characteristics length and time depend on the studied system. However, the comparison between very different systems like pure fluids, binary liquid mixtures, polymers blends, micellar systems, microemulsions, alloys and inorganic glasses is made difficult by the use of many different characteristics scales. Besides, the conditions in which the phase separation dynamics occurs, are not very well defined in most of these experiments. Moreover, the interaction between growing domains as well as the coupling with an external field (like gravity for instance) strongly disturbs the 
analysis of the data. In the first part of this communication, we present the results of an original experiment of laser-induced local concentration variations in order to drive a phase separation in a micellar phase of microemulsion. This laser constraint allows the study of the growth of an isolated beam trapped droplet. The trapping results in a significative reduction of the gravitational coupling. In the second part, we compare this dynamical behavior with that of other systems belonging to the same Ising class $d=3$ and $n=1$, using the same scaling.

\section{DYNAMICS OF LASER-INDUCED PHASE SEPARATION IN A MICELLAR PHASE OF MICROEMULSION.}

Since the pioneer work of Ashkin [14] on trapping and levitation of particles with a strongly focused laser beam, it has been shown that laser waves are able to locally modulate the particle density of suspensions and hence, to change the composition of any mixture whose components have different refractive indices. Physically, two different mechanisms account for such concentration variations [15]. The first one, called electrostriction, is due to electrostrictive forces which originate from the coupling of the dipole moment induced by the field on each particle, with the gradient of this field. These dipolar forces are proportional to the particle polarizability and to the intensity gradient. Besides, although we consider off-resonance optical fields, a suspension is always characterized by a small residual absorption at the used wavelength. Due to the gaussian nature of laser waves, a weak beam-centered thermal gradient is generated. Even small, it can induce a noticeable concentration gradient due to thermodiffusion (also called Soret effect) [15]. In the following, we use these laser-induced electrostrictive and thermodiffusive concentration variations to drive a phase separation in a binary liquid mixture.

The laser-induced quench in composition is analyzed in a micellar phase of microemulsion of water, $n$-dodecane, sodium dodecyl sulfate and $n$-pentanol. In the rich oil part of the phase diagram, the mixture features a micellar structure: this is a stable suspension of very small droplets of water, called micelles (radius $=40 \AA$, micellar fraction $=11 \%$ ), isolated from the continuous oil phase by a shell of soap and alcohol molecules. For the chosen off-critical composition of the mixture [16], above the critical temperature, the system separates into two micellar phases: one of richer micellar concentration $\left(c_{1}\right)$ than the other $\left(c_{2}\right)$. The density of the phase of concentration $c_{1}$ is larger than that of concentration $c_{2}$. Figure 1 a) describes schematicaly the phase diagram in the plane $(c, T)$.

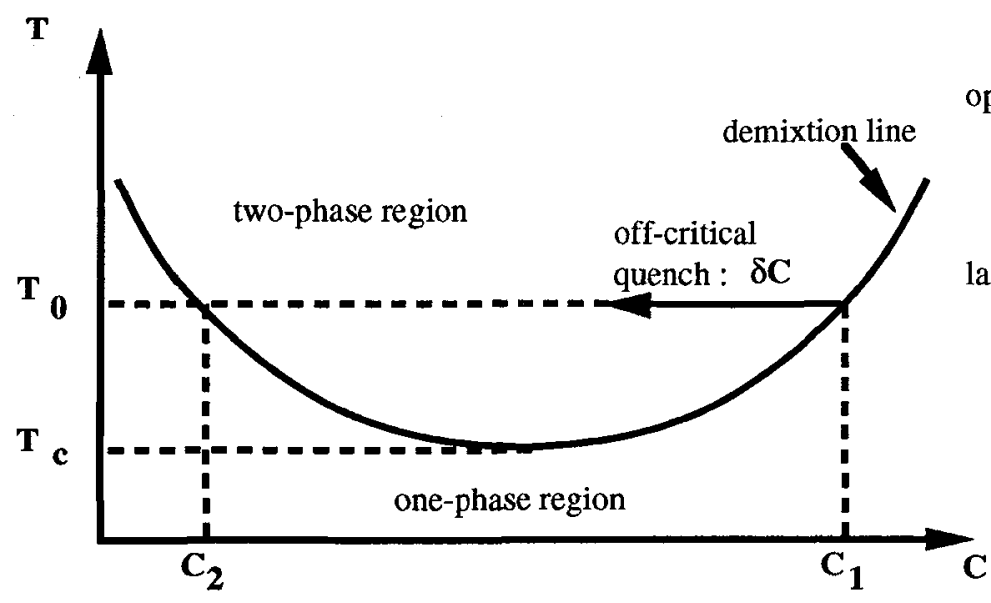

a)

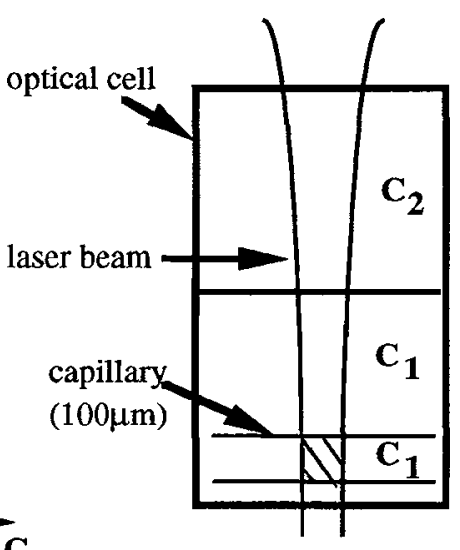

b)

figure 1: a) the schematic phase diagram where $\delta \mathrm{c}$ represents the isothermal optical quench in the metastable region. $b$ ) experimental configuration (the interaction volume is $\approx 2.10^{-8}$ of the total volume of the phase of concentration $c_{1}$ ).

The initial motivation of this choice of micellar phase of microemulsion has basically been two fold. Firstly, due to the supramolecular size of the micelles, microemulsions seem to be very attractive 
for analyzing the dynamics of phase separation [17]. Secondly, they are characterized by a large sensitivity to laser waves. On the one hand, the size of micelles gives them a very large polarizability compared to that of molecules in classical binary liquid mixtures. On the other hand, these systems are characterized by a weak absorption in the visible domain (of the order of a few $10^{-4} \mathrm{~cm}^{-1}$ ) and by an important thermodiffusion ratio, such that a very small increase of temperature can result in an important variation of the concentration. Then, both electrostrictive and thermodiffusive processes lead to a measurable micellar concentration variation on the laser beam axis (in our case a decrease of the micellar concentration) and thus induce a phase separation in a sample of concentration $c_{1}$. Moreover, since the temperature increase in the mixture is weak and the interaction volume is small compared to the total volume (see figure $1 \mathrm{~b}$ )), this phase transition can be considered as an isothermal process.

Such an experiment has been performed by focusing with a x10 microscope objective a c.w. $\mathrm{Ar}^{+}$ laser beam in a thermally controlled optical cell. The phase separation is analyzed with a C.C.D. video camera coupled to an image analysis system. As soon as the field is applied, the phase of concentration $c_{1}$ is locally quenched (due to the $\delta c$ concentration variation) in the metastable region (figure $1 \mathrm{a}$ )). As a result, droplets constituted by the new phase $\left(c_{2}\right)$ are nucleated in the laser beam [18]. These droplets are characterized by a refractive index $n_{2}$ larger than that of the surrounding phase $\left(c_{1}\right) n_{1}$, since the refractive index of water is smaller than that of oil. Their polarizability is then positive and the electrostrictive coupling due to the field gradient moves them towards the beam axis. The resulting optical trapping in the cell (10mm thick) is illustrated in the figure 2. However, the late stage of the growth is modified by gravity.

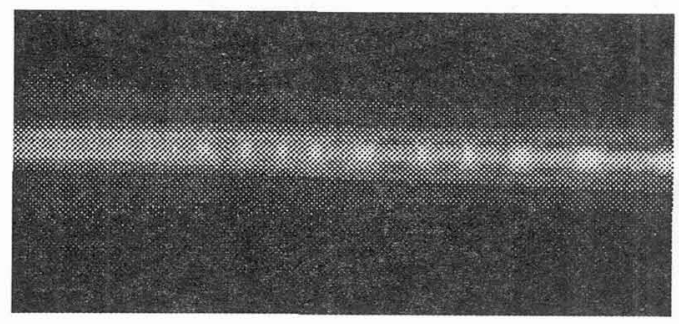

figure 2:1aser-induced phase separation in the cell (10 mm thick) [18]
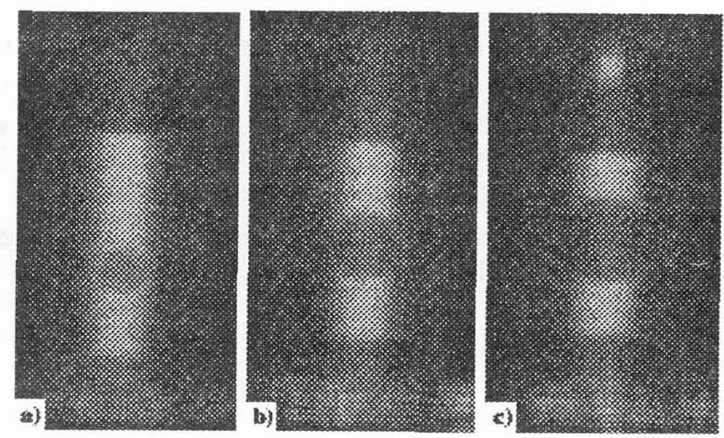

figure 3:laser-induced phase separation in the capillary $(100 \mu \mathrm{m}), \mathrm{a}) \mathrm{t}=300 \mathrm{~s}, \mathrm{~b}) \mathrm{t}=600 \mathrm{~s}, \mathrm{c}) \mathrm{t}=960 \mathrm{~s}$ (see figure $2 \mathrm{~b}$ ): $2 \mathrm{~mm}$ thick for optical cell)

In fact, the study of fundamental growth mechanisms would consist in the analysis of the evolution of a single droplet in compensed-gravity. With this aim in mind, we realized the same experiment in a very weak optical path capillary $(100 \mu \mathrm{m})$, which is set at the bottom of the cell and is thus dipped in the phase of concentration $\mathrm{c}_{1}$ (figure $1 \mathrm{~b}$ )). After an optical quench in this configuration, a selection mechanism leads to three beam trapped droplets. Two among them wet walls of the capillary on both sides of a central one (figure 3). This last is transversely and longitudinaly trapped, respectively, by electrostriction and by repulsive interactions induced by the two others droplets. These interactions completely dominate gravity. Thus, this experiment in which a laser wave acts as an optical tweezer, allows the analysis of the dynamics of a single droplet in the diffusion regime over very large time scales. In the following, we analyze this growth in the frame of the phase separation dynamics inside the universality class $\mathrm{d}=3$ and $\mathrm{n}=1$. 
3.ANALYSIS IN REDUCED LENGTH AND TIME SCALES FOR PHASE SEPARATION DYNAMICS IN THE ISING CLASS $(d=3, n=1)$

When a whole system is brought from the one-phase region to the two-phase region, the orderparameter fluctuations become unstable and lead to phase separation. This phase transition may proceed either by droplet nucleation (metastable region) or by spinodal decomposition (unstable region). The latter case is characterized by a pattern of interconnected domains that interact continuously. Their characteristic size is $\mathrm{L}_{\mathrm{m}}(\mathrm{t})=2 \pi / \mathrm{k}_{\mathrm{m}}(\mathrm{t})$ [2]. This expression is the usual experimental definition of $\mathrm{L}_{\mathrm{m}}$ where $\mathrm{k}_{\mathrm{m}}$ is the peak position of the maximum scattered intensity $I(k, t)$ [1]. $\mathrm{L}_{m}(t)$ grows as $t^{\mathrm{a}}$ where $t$ is the time and $\mathrm{a}$ is an exponent evolving as phase separation proceeds. The dynamics of phase separation of a critical system may be simplified into three mains regimes associated to three exponent values: the early stage also known as the Cahn regime $(a \approx 0)$, the intermediate stage controlled by diffusion mechanisms $(a \approx 1 / 3)$ and the late stage dominated by the hydrodynamic interactions $(a \approx 1)$.

Because of the large differences in length and time scales observed in real systems, the scaling of their dynamical properties requires dimensionless reduced variables. Classically, two reduced parameters defined as $\mathrm{Qm}(\tau)=\mathrm{k}_{\mathrm{m}}(\mathrm{t}) / \mathrm{k}_{\mathrm{m}}(0)$ and $\tau=\mathrm{t} / \mathrm{t}_{\mathrm{c}}$ are sufficient. $\left(\mathrm{k}_{\mathrm{m}}(0)\right)^{-1}$ is the characteristic length of the studied system and $\mathrm{t}_{\mathrm{c}}$ the characteristic time defined by $\left(\mathrm{t}_{\mathrm{c}}\right)^{-1}=\mathrm{D} *\left(\mathrm{k}_{\mathrm{m}}(0)\right)^{2}$, where $\mathrm{D}$ is a mass diffusion coefficient precised in table 1.

The first experiments performed on some systems of the same molecular nature for different quench depths have attempted to show that the evolution of $\mathrm{k}_{\mathrm{m}}(\mathrm{t})$ could be reported on an universal curve where $\mathrm{Q}_{\mathrm{m}}^{-1}->1$ when $\tau-->0$ [3]. Besides, H.L. Snyder and P. Meakin [4] have compared experimental results in small molecule liquid mixtures, high molecular weight polymers, inorganic glasses and metallic alloys to determine if there was a common dynamical behavior in these widely differing systems. Indeed all the results seemed to collapse on a single curve $\mathrm{Qm}_{m}(\tau)$ for $\tau->0$, with the same asymptotic limit. Moreover, their analysis have suggested that in spite of the wide differences between these systems, a similar dynamic governs phase separation processes. They finally concluded that further experimental works would be necessary to confirm their conjecture. In order to more precisely analyze this suggested scaling behavior, Furukawa proposed in 1985, the following analytic function for $\mathrm{Q}_{\mathrm{m}}(\tau)$ available to the early-to-late stage of spinodal decomposition [5].

$$
\left(\mathrm{Q}_{\mathrm{m}}^{-1}-1-\left(\left(\frac{\mathrm{A}^{\prime}}{\mathrm{B}^{\prime}}\right)^{1 / 2} *\left[\arctan \left(\mathrm{Q}_{\mathrm{m}}^{-1} *\left(\frac{\mathrm{B}^{\prime}}{\mathrm{A}^{\prime}}\right)^{1 / 2}\right)-\arctan \left(\frac{\mathrm{B}^{\prime}}{\mathrm{A}^{\prime}}\right)^{1 / 2}\right]\right)\right)=\mathrm{B}^{\prime} * \tau
$$

This expression has been obtained by integration of equation (2) using the boundary condition $\mathrm{Q}_{\mathrm{m}}^{-1}->1$ when $\tau->0$ :

$$
\frac{\mathrm{dQ}_{\mathrm{m}}^{-1}}{\mathrm{~d} \tau}=\mathrm{A}^{\prime} \mathrm{Q}_{\mathrm{m}}^{2}+\mathrm{B}^{\prime}
$$

The equation (1) can describe the two behaviors $\mathrm{Qm}_{\mathrm{m}}(\tau) \approx(\tau)^{-1 / 3}$ and $\mathrm{Qm}_{\mathrm{m}}(\tau) \approx(\tau)^{-1}$ choosing the correct values for parameters $A^{\prime}$ and $B^{\prime}$. The value of $A^{\prime}$ is estimated in order to recover the behavior in the early and intermediate stage predicted by Kawasaki and Ohta [6], while the value of B' is evaluated to recover the late stage dynamic according to Siggia's procedure [7]. In fact, the situation is still much more complicated because $\mathrm{k}_{\mathrm{m}}(0)$ and $\mathrm{D}$ are difficult to measure for each studied system. So different characteristics length and time have been used in the litterature to obtain this reduced dynamical behavior. In order to show these puzzling choices, we have reported on table 1 the corresponding definitions of $\mathrm{k}_{\mathrm{m}}(0)$ and $\mathrm{D}$ for some subsystems belonging to $d=3, n=1$ universality class. 


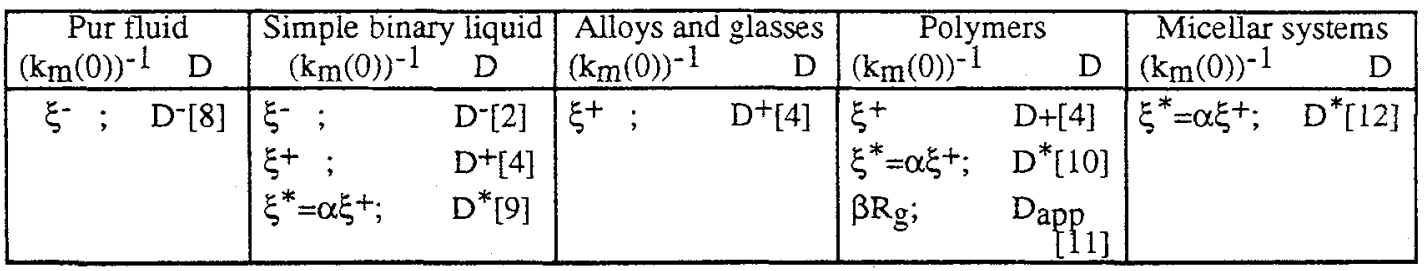

table 1: expressions of $\left(\mathrm{k}_{\mathrm{m}}(0)\right)^{-1}$ and $\mathrm{D}$ for systems of universality class, where $\xi^{+}$and $\xi^{-}$are respectively, the correlation length in the one and the two phase region, $\mathrm{R}_{\mathrm{g}}$ the gyration radius, $\mathrm{D}^{+}$and $\mathrm{D}^{-}$are respectively the diffusion coefficient in the one and the two-phase regions, $D^{*}$ the interdiffusion coefficient and $\mathrm{D}_{\text {app }}$ the collective diffusion coefficient.

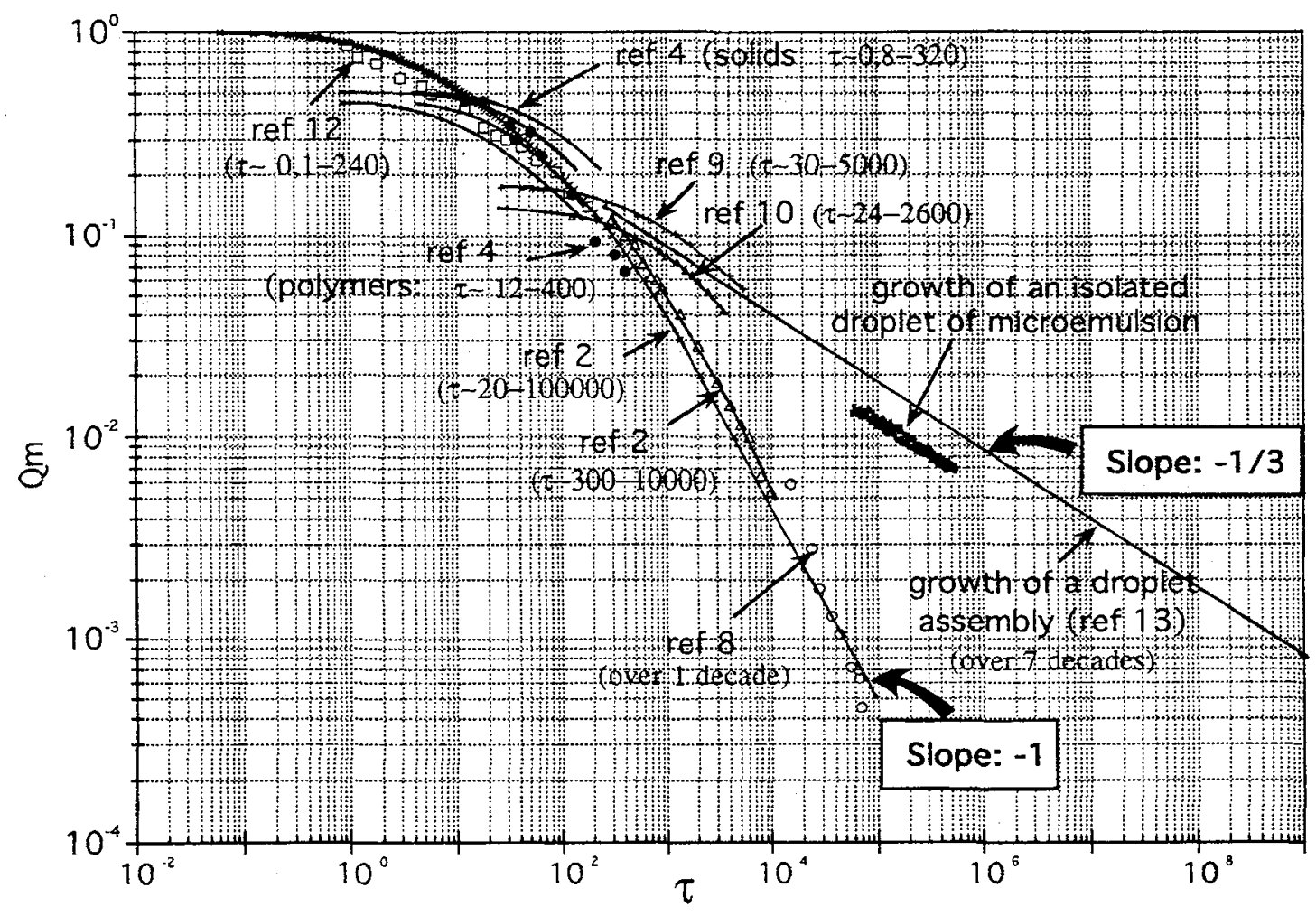

figure 4: Comparison of reduced experimental results of systems referenced in table 1, using the peculiar choice $\mathrm{Qm}=\mathrm{k}_{\mathrm{m}}(\mathrm{t}) \xi^{-}$and $\tau=\mathrm{D}^{-} \mathrm{t}\left(\xi^{-}\right)^{2}$ of the reference [13]. The two late stages characterized by slopes -1 and $-1 / 3$ are discussed below. 
Using $\mathrm{Qm}_{\mathrm{m}}=\mathrm{k}_{\mathrm{m}}(\mathrm{t}) \xi^{-}$and $\tau=\mathrm{D}^{-} \mathrm{t} /\left(\xi^{-}\right)^{2}$ [13], the results obtained in the critical case (characterized by an equilibrium volume fraction of the minority phase $\phi=1 / 2$ ) of both pure fluids [8] and binary liquid mixtures [2] have shown a pattern with interconnected domains associated to a late stage scaled behavior $\mathrm{Qm}(\tau) \approx(\tau)^{-1}$.

Besides, in the case of off-critical systems $(\phi<<0.5)$, a phase separation may also proceed by droplet growth, i.e. with a morphology completely different. Recently, experiments in binary liquids and pure fluids have also been realized in this last case for $0.1<\phi<0.4$ [13]. A morphology of a tightly packed droplets have been observed. Moreover, the analysis with the above mentioned definitions of $\mathrm{Qm}$ and $\tau$ have pointed out a second late stage scaled behavior $\mathrm{Qm}(\tau) \approx(\tau)^{-1 / 3}$.

To show the relative position in relation to these two scaling laws, we have rescaled all the systems presented in table 1 , using this peculiar choice of $\mathrm{Qm}$ and $\tau$. The resulting scalings for these systems are reported in figure 4. In spite of an important dispersion of experimental results, it appears on this figure that the experimental behavior $\mathrm{Qm}_{\mathrm{m}}(\tau) \approx(\tau)^{-1 / 3}$ described by F. Perrot et al. [13] is the slowest for all these systems for which the morphology is either interconnected domains or droplets.

In our particular case of laser-induced off-critical phase separation in microemulsion, the growth of the central beam-trapped droplet is $\mathrm{R} \approx \mathrm{t}^{1 / 3}$. Our results for three quenches in composition are also reported on figure 4. They suggest that the reduced growth law of this droplet is comparable to that of a tightly packed droplets in microgravity. In fact, our data are slightly below this limit, certainly due to coalescence mechanism between a few droplets in early time.

In conclusion, this technique allows to analyze the growth of an isolated droplet in a well-defined configuration. It also opens a new field in the control and the discrimination of various processes involved in phase separation. To obtain a reduced behavior, we have scaled this growth in the general context of the phase separation kinetics inside the universality class $d=3$ and $n=1$ of the Ising model. This study suggests that the experimental behavior $\mathrm{Qm}_{\mathrm{m}}(\tau) \approx(\tau)^{-1 / 3}$ describes the slowest dynamics at the late stage.

\section{REFERENCES:}

Book : [1]: J. D. Gunton, M. San Miguel, and P. S. Sahni, in Phase Transition and Critical Phenomena, edited by C. Domb and J. L. Lebowitz (Academic, New York, 1983), Vol. 8.

Periodicals:[2]: P. Guenoun, R. Gastaud, F. Perrot, and D. Beysens, Phys. Rev. A 36 (1987)4876.

[3]: Y. C. Chou and W. I. Goldburg, Phys. Rev. A 20 (1979) 2105.

[4]: H. L. Snyder and P. Meakin, J. Chem. Phys. 79 (1983) 5588.

[5]: H. Furukawa, Adv. Phys. 34 (1985) 703.

[6]: K. Kawasaki and T. Ohta, Progr. theor. Phys. 59 (1978) 362.

[7]: E. D. Siggia, Phys. Rev. A 20 (1979) 595.

[8]: Y. Garrabos, B.Le Neindre, P. Guenoun, B. Khalil, D. Beysens, Europhys. Lett. 19 (1992) 491.

[9]: N. Kuwahara, K. Kubota, M.Sakazume, H. Eda, and K. Takiwaki, Phys. Rev. A 45 (1992) 8324.

[10]: N. Kuwahara and K. Kubota, Phys. Rev. A 45 (1992) 7385.

[11]: F. S. Bates and P. Wiltzius, J. Chem. Phys. 91 (1989) 3258.

[12]: N. Kuwahara and K. Kubota, Phys. Rev. A 46 (1992) 6501.

[13]: F. Perrot, P. Guenoun, T. Baumberger, D. Beysens, Y. Garrabos and B. Le Neindre, Phys. Rev. Lett. 73 (1994) 688.

[14]: A. Ashkin, Phys. Rev. Lett. 24 (1970) 156.

[15]:B. Jean-Jean, E. Freysz, A. Ponton, A. Ducasse, and B. Pouligny, Phys. Rev. A 39 (1989). 5268

[16]: D. Gazeau, E. Freysz, and A.M. Bellocq, Europhys. Lett. 9 (1989) 833.

[17]:D. Roux, J. Physique 47 (1986) 733

[18]:J.P. Delville, C. Lalaude, E. Freysz, and A. Ducasse, Phys. Rev. E 49 (1994) 4145 